\title{
An Accurate and Comprehensive Soft Error Simulator NISES II
}

\author{
Y. Tosaka, S. Satoh, and H. Oka \\ Fujitsu Laboratories Ltd., \\ 50 Fuchigami, Akiruno, Tokyo 197-0833, Japan \\ tosaka@flab.fujitsu.co.jp
}

\begin{abstract}
The authors report an accurate and comprehensive soft error (SE) simulator NISES version II, which includes effects due to cosmic ray high-energy neutrons, thermal neutrons, and $\alpha$ - particles. NISES II covers SE analysis of DRAM, SRAM, and latch (or FF) circuits and it is also applicable for SE analysis of SOI circuits.
\end{abstract}

\section{Introduction}

As the device density increased in VLSI, the SE has become a serious problem especially for SRAM and logic circuits [1]. SE simulations are indispensable to estimate soft error rates (SERs) at the circuit design stage and an accurate and comprehensive SE simulator is strongly requested. We report an SE simulator NISES (Neutron-Induced Soft Error Simulator) version II, which is the most comprehensive one among SE simulators previously reported [2-6].

NISES was originally developed for analysis of secondary cosmic ray high-E neutroninduced SEs [3,4]. In NISES II, an accuracy of SER estimations is remarkably improved and, at the first time, it includes effects of thermal neutrons as well as highE neutrons and $\alpha$-particles, and also includes bipolar effects of SEs in SOI circuits. NISES II covers SE analysis for DRAM, SRAM, and latch (or FF) circuits.

\section{Outline of NISES II}

The sources of SEs are high-E neutrons, thermal neutrons, and $\alpha$-particles (Fig. 1) $[1,7]$. High-E neutrons and thermal neutrons induce SEs through neutron-nucleus reactions in the silicon substrate and thermal neutron capture reactions of ${ }^{10} \mathrm{~B}$ in BPSG (borophosphosilicate glass), respectively.

Figure 2 shows the outline of NISES II. The device structure is the input data. Neutron-silicon reactions, ${ }^{10} \mathrm{~B}(\mathrm{n}, \alpha){ }^{7} \mathrm{Li}$ reactions, and $\alpha$-particles are randomly generated in the silicon substrate, BPSG, and source materials using a Monte Carlo procedure. If the charged particle passes through a charge-collected region, the charges induced in the region are calculated. We assumed that when these charges pass a critical charge Qc, an SE occurs.

The nuclear reaction database for high-E neutron-induced SEs, was updated by using AMD-V [8], which is modified version of AMD (Antisymmetrized Molecular Dynamics). Estimation method for dimensions of the charge-collected region and for Qc were improved by using device and circuit simulations. Bipolar effects of SEs in SOI circuits were also included in NISES II. 


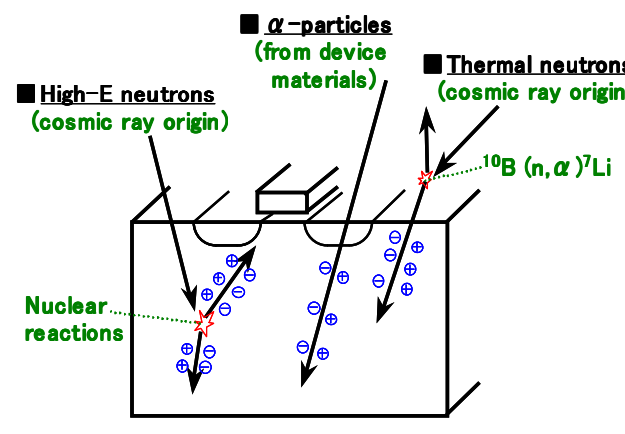

Figure 1: Sources of soft errors.

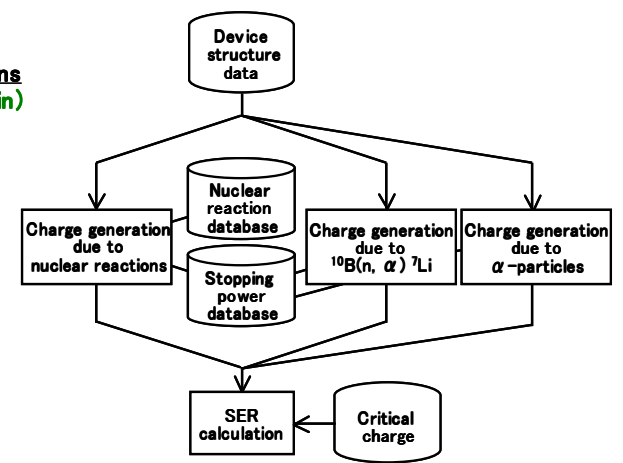

Figure 2: Outline of NISES II.

\section{Applications}

Accurate estimations of multiple-bit SEs are important for the ECC (Error Correction Code) design [9]. Figure 3 shows high-E neutron-induced multiple-bit SEs in $16 \mathrm{Mb}$ DRAMs. Results of NISES version I and version II ware compared with data measured at Los Alamos National Laboratories using a pulsed neutron beam, which has an energy spectrum similar to that of atmospheric neutrons. Accuracy of multiplebit SERs was remarkably improved, because accuracy of nuclear reaction data for heavy recoil ions is improved in new database. The simulated SERs agreed with measured SERs within a factor of 2 for all multiplicity.

Figure 4 and 5 show accelerated $\alpha$ - and neutron- SERs in SRAM with $0.13 \mu \mathrm{m}$ technology. $\alpha$-accelerated tests were performed using ${ }^{241} \mathrm{Am}$ and neutron-accelerated tests were performed at RCNP (Research Center for Nuclear Physics, Osaka Univ.), using a $135 \mathrm{MeV}$ quasi-mono energy neutron beam. Simulated SERs agreed with measured SERs within 20-40\% errors. An accuracy of SER estimations of the original version of NISES was within a factor of 2-5 [4]. Therefore, the accuracy is improved in NISES II.

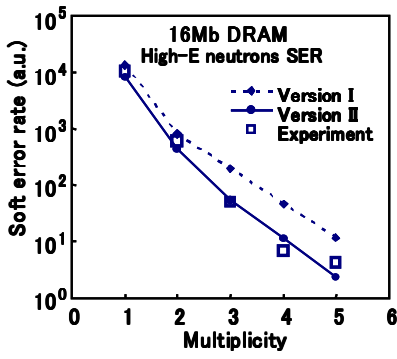

Figure 3: High-E neutron induced multiple-bit SERs in $16 \mathrm{Mb}$ DRAM.

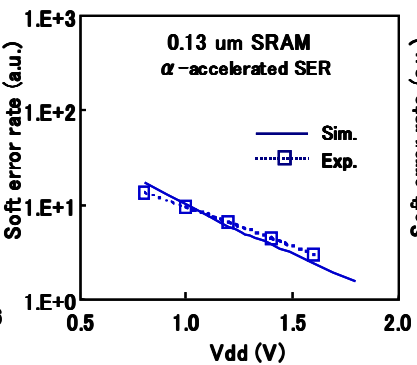

Figu re 4: $\alpha$-accelerated SERs in $0.13 \mu \mathrm{m}$ SRAM.

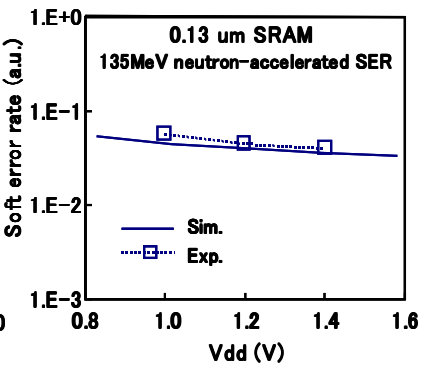

Figure 5: $135 \mathrm{MeV}$ neutron-accelerated SERs in $0.13 \mu \mathrm{m}$ SRAM. 
Figure 6 shows simulated ratio of SERs in $0.18 \mu \mathrm{m}$ SRAM with BPSG. About 87\% of SER is due to thermal neutrons. However, because the CMP process is used in place of BPSG in recent CMOS technologies, we can ignore the effect of thermal neutrons in most cases. Figure 7 shows the estimated SER trend in SRAMs as a function of technology node. We assumed an atmospheric neutron spectrum and an $\alpha$-emission rate of $0.001 / \mathrm{cm}^{2} /$ hour (typical value for material, for examples, mold compound, low- $\alpha \mathrm{SnAg}$ bump and etc.). Currently, SERs due to $\alpha$-particles are similar to those due to high-E neutrons. The SRAM SERs for single cell have similar values in recent technologies, which is consistant with ref. [1].

Because SOI devices are candidate for the solution in future VLSI, SE simulations for SOI circuits are important. When a charged particle hits the channel region in an SOI MOSFT, collected charges remarkably increases due to bipolar effects (Fig.8) [10].

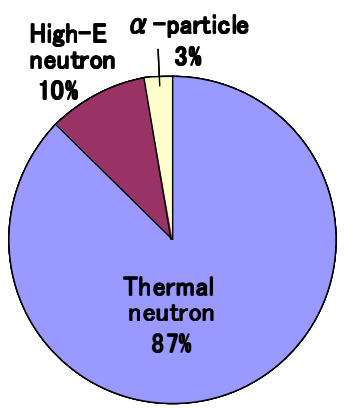

Figure 6: Simulated ratio of SERs in $0.18 \mu \mathrm{m}$ SRAM with BPSG.

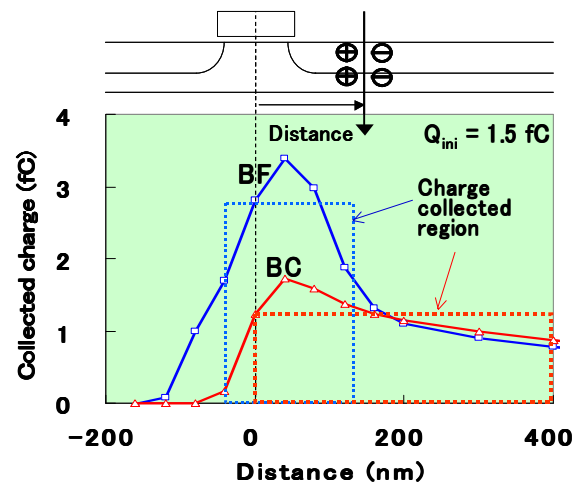

Figure 8: Bipolar effect induced by a charged particle and the chargecollected region in an SOI MOSFET.

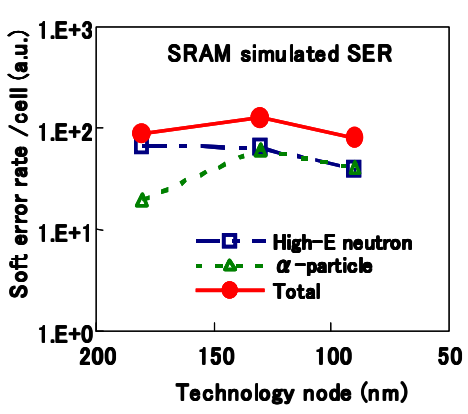

Figure 7: SER trend in SRAMs.

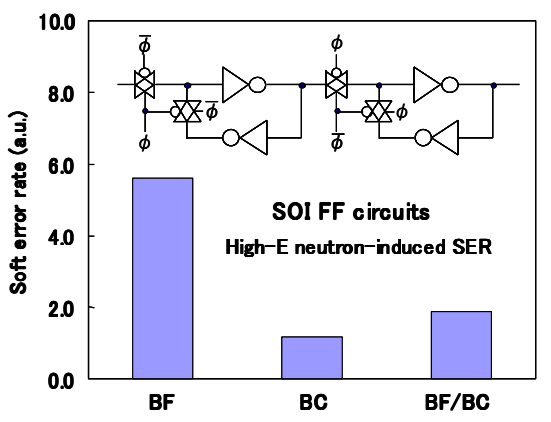

Figure 9: High-E neutron-induced SERs in SOI FF circuits. 
A charge-collected region is determined by using device simulations and critical initial charge $\mathrm{Q}_{0} \mathrm{c}$ (charges induced in the channel region, $\mathrm{Q}_{0} \mathrm{c}<\mathrm{Qc}$ ) is determined by combination with device and circuit simulations. Figure 9 shows high-E neutron induced SER in SOI latch (or FF) circuit. SER with floating body (FB) structure is larger than that with body contact (BC) due to bipolar effect. SER with FB/BC mixed structure is slightly larger than that with $\mathrm{BC}$. $\mathrm{FB} / \mathrm{BC}$ mixed structure is one of solutions to decrease both SER and cell area.

\section{Summary}

The most comprehensive SE simulator NISES II includes effects of thermal neutrons as well as high-E neutrons and $\alpha$-particles, and also includes bipolar effects of SEs in SOI circuits. NISES II covers a wide range of SE analysis for DRAM, SRAM, and latch circuits with high accuracy.

\section{Acknowledgement}

The authors thank A. Ono of Tohoku Univ. and H. Horiuchi of Kyoto Univ. for suggestions about AMD-V; S. A. Wender of Los Alamos National Lab., K. Hatanaka of RCNP, H. Ehara and K. Takasu of Fujitsu for their assistance in experiments.

\section{References}

[1] R. Baumann, "The impact of technology scaling on soft error rate performance and limits to the efficacy of error correction", IEEE Proc. 2002 IEDM, pp. 329-332.

[2] G.R. Srinivasan, P.C. Murey, and H.K. Tang, "Accurate, predictive modeling of soft error rate due to cosmic rays and chip alpha radiation", IEEE Proc. 1994 IRPS, pp. 12-16.

[3] Y. Tosaka, S. Satoh, and T. Itakura, "Neutron-induced soft error simulator and its accurate predictions, IEEE Proc. 1997 SISPAD, pp.253-256.

[4] Y. Tosaka, H. Kanata, S. Satoh, and T. Itakura, "Simulation technologies for cosmic ray neutron-induced soft errors: Models and simulation systems"., IEEE Trans. Nucl. Sci., Vol. 46, pp.774-780, 1999.

[5] M. Hane, Y. Kawakami, H. Nakamura, T. Yamada, K. Kumagai, and Y. Watanabe, "A new comprehensive SRAM soft-error simulation based on 3D device simulation incorporateing neutron neuclear reactions" IEEE Proc. 2003 SISPAD, p.239-242.

[6] E. Ibe, Y. Yahagi, F. Kataoka, Y. Saito, A. Eto, M. Sato, H. Kameyama and M. Hidaka, "A self-consistant integrated system for terrestrial-neutron induced single event upset of semiconductor devices at the ground level”, 2002 ICITA, Bathurst, No273-21.

[7] H. Kobayashi, K. Shiraishi, H. Tsuchiya, M. Motoyoshi, H. Usuki, Y. Nagai, K. Takahisa, T. Yoshiie, Y. Sakurai, and T. Ishizaki, "Soft errors in SRAM devices induced by high energy neutrons, thermal neutrons and alpha particles", IEEE Proc. 2002 IEDM, p.337340.

[8] Y. Tosaka, A. Ono, and H. Horiuchi, "Nucleon-induced fragment formation with antisymmetrized molecular dynamics", Phy. Rev. C, Vol. 60, pp.064613-064621, 1999.

[9] Y. Tosaka and S. Satoh, "Simulation of multiple-bit soft errors induced by cosmic ray neutrons in DRAMs", IEEE Proc. 2000 SISPAD, pp.265-268.

[10] Y. Tosaka, K. Suzuki, and T. Sugii, "Alpha-particle-induced soft errors in submicron SOI SRAM”, 1995 VLSI Tech. Dig., pp.39-40. 